\title{
Estimating the mean effect size in meta-analysis: Bias, precision, and mean squared error of different weighting methods
}

\author{
WIM VAN DEN NOORTGATE and PATRICK ONGHENA \\ Katholieke Universiteit Leuven, Leuven, Belgium
}

\begin{abstract}
Although use of the standardized mean difference in meta-analysisis appealing for severalreasons, there are some drawbacks. In this article, we focus on the following problem: that a precision-weighted mean of the observed effect sizes results in a biased estimate of the mean standardized mean difference. This bias is due to the fact that the weight given to an observed effect size depends on this observed effectsize. In order to eliminate the bias, Hedges and Olkin (1985) proposed using the mean effect size estimate to calculate the weights. In the article, we propose a third alternative for calculating the weights: using empiricalBayes estimates of the effect sizes. In a simulation study, these three approaches are compared. The mean squared error ( $M S E$ ) is used as the criterion by which to evaluate the resulting estimates of the mean effect size. For a meta-analytic dataset with a small number of studies, the MSE is usually smallest when the ordinary procedure is used, whereas for a moderate or large number of studies, the procedures yielding the best results are the empirical Bayes procedure and the procedure of Hedges and Olkin, respectively.
\end{abstract}

The standardized mean difference was first used by Cohen (1969) to perform power calculationsfor $t$ tests. Ever since, this measure of effect size has frequently been used to summarize the results of group comparison studies. Glass and his colleagues (Glass, 1976, 1977; Glass, MacGaw, \& Smith, 1981), who were among the first to present a systematic set of meta-analytic techniques, suggested using this measure in meta-analysis. The sampling distribution of the effect size measure and the statistical background for its use in meta-analysis was provided by Hedges (1981, 1982). Although other measures of effect size have been advocated in meta-analytic research-for instance, the correlation coefficient (e.g., Hunter, Schmidt, \& Jackson, 1982) or Fisher's $Z$ (e.g., Rosenthal, 1991)—the standardized mean difference still plays a prominent role in metaanalytic research.

In this article, we discuss a problem that shows up when the overall or mean standardized mean difference is estimated with standard methods. Traditionally, the grand mean effect is usually estimated by a weighted average of the observed effects. The precision of an observed effect size, which is the inverse of the sampling variance, is often used to weight an observed effect size. Owing to the dependence of the weights and the (observed) standardized mean difference, the estimate of the mean standardized mean difference will be biased. As we shall see below, several alternative estimation procedures are possible, but from the literature it is not clear whether there is a uniformly best

Correspondence concerning this article should be addressed to W. Van den Noortgate, Department of Education, Katholieke Universiteit Leuven, Vesaliusstraat 2, B-3000Leuven, Belgium (e-mail: wim.vandennoortgate@ ped.kuleuven.ac.be). approach or in which situation one or another approach is to be preferred. One appealing approach has been proposed by Hedges and Olkin (1985). They proposed making the weights independent of the observed effect sizes by using the overall effect size estimate to estimate the weights. Recent developments in meta-analytic literature, however, suggest that empirical Bayes estimates of the study effect sizes are optimal estimates of the study effect sizes, reducing the mean squared error (MSE; Raudenbush \& Bryk, 1985). Hence, a plausible alternative approach for estimating the sampling variances of the observed effect sizes and the corresponding weights, which however has not been proposed before, is to use the empirical Bayes estimates of the study effect sizes. The purpose of this article is (1) to explore the extent of the problem of bias, (2) to present the empirical Bayes approach for estimating the weights, and (3) to evaluate the mean effect size estimates based on the ordinary procedure, the empirical Bayes procedure, and the overall effect size procedure for estimating the weights. We will start with a short description of the standardized mean difference and of common meta-analytic techniques. Next, we will discuss the problem of estimating the grand mean effect size when standard methods are used and will present some potential improvements of the estimation procedure. Three alternatives will be compared, using a small simulation study. We will finish with some conclusions and recommendations.

\section{The Standardized Mean Difference}

The standardized mean difference, $\delta$, is defined as the difference between two population means divided by the standard deviation of one of both populations or by a common population standard deviation $(\sigma)$. It is often used 
when an experimental population is compared with a control population, in order to investigate the effect of a certain intervention:

$$
\delta=\frac{\mu_{\mathrm{E}}-\mu_{\mathrm{C}}}{\sigma},
$$

where $\mu_{\mathrm{E}}$ and $\mu_{\mathrm{C}}$ the means of the experimental and the control populations, respectively.

This measure is sometimes called Cohen's $d$, but because it is a population parameter, we prefer using the Greek symbol $\delta$. Hedges and Olkin (1985) argued that in experimental research, it is often sensible to assume a common population variance. An unbiased estimate of this variance can be obtained by pooling the two sample estimates:

$$
s_{\mathrm{p}}^{2}=\frac{\left(n_{\mathrm{E}}-1\right) s_{\mathrm{E}}^{2}+\left(n_{\mathrm{C}}-1\right) s_{\mathrm{C}}^{2}}{N-2},
$$

where $n_{\mathrm{E}}$ is the size of the experimental group, $n_{\mathrm{C}}$ is the size of the control group, and $N$ is equal to $n_{\mathrm{E}}+n_{\mathrm{C}}$.

An obvious estimate of $\delta$, therefore, is its sample counterpart $g$ :

$$
g=\frac{X_{\mathrm{E}}-X_{C}}{s_{\mathrm{p}}} .
$$

Although the sample mean difference is an unbiased estimator of the population mean difference and the pooled variance estimate is an unbiased estimate of a common population variance, $g$ is a biased estimator of $\delta$, especially for smaller studies (Hedges, 1981). An unbiased estimator $d$ can be obtained by multiplying $g$ by a correction factor. Hedges (1981) found that $d$ is very closely approximated by

$$
d \cong g \cdot[1-3 /(4 N-9)] .
$$

Hedges (1981) showed that if both the experimental and the control population distributions are normal and $n_{E}$ and $n_{C}$ are moderate to large (at least equal to 10 ), the sampling distribution of $d$ is approximately normal:

$$
d \sim N\left[\delta, \sigma^{2}(d)\right],
$$

where

$$
\sigma^{2}(d) \cong \frac{N}{n_{E} n_{C}}+\frac{\delta^{2}}{2 N} .
$$

The sampling variance depends on the unknown population parameter $\delta$ but is closely approximated by replacing $\delta$ in Equation 5 by the estimate of $\delta$ :

$$
d \sim N\left[\delta, \hat{\sigma}^{2}(d)\right],
$$

where

$$
\hat{\sigma}^{2}(d)=\frac{N}{n_{\mathrm{E}} n_{\mathrm{C}}}+\frac{d^{2}}{2 N} .
$$

\section{Combining Effect Size Estimates From Different Studies}

In meta-analytic research, it is often assumed that, in each study, the same effect size $\delta$ is estimated. This $\delta$ is estimated by averaging the observed effect sizes. Because studies often differ in size and, therefore, the precision of the estimates differs, usually a weighted mean of the observed effect sizes is calculated. By using weights equal to the precision (the inverse of the variance calculated using Equation 6), the variance of $\hat{\delta}$ is reduced (e.g., Hedges, 1982):

$$
\hat{\delta}=d_{+}=\frac{\sum_{i=1}^{k} w_{j} d_{j}}{\sum_{j=1}^{k} w_{j}},
$$

where $w_{j}=1 / \hat{\sigma}^{2}\left(d_{j}\right)$ and $k=$ the number of studies.

Often, however, the assumption of homogeneous studies (i.e., studies in which a common effect size is estimated) is questionable, because studies usually are not exact replications of each other. Although in this case the fixed effects procedure presented above is still appropriate if the true effects investigated in the set of studies are regarded as the effects of interests (Hedges \& Vevea, 1998), a random effects meta-analytic model may be more appropriate. In a random effects meta-analytic model, the true effect sizes in a set of studies are viewed as a random sample out of a population of effect sizes (Rubin, 1981). Consequently, in this model, there are two sources of variation of observed effect sizes: variation between true effect sizes and sampling variation for each study. Because these two sources are independent,

$$
\sigma^{2}\left(d_{j}\right)=\sigma^{2}(\delta)+\sigma^{2}\left(d_{j} \mid \delta_{j}\right) .
$$

Meta-analytic procedures for random effects models (REMs) were further developed by Hedges (1983; Hedges \& Olkin, 1985) and DerSimonian and Laird (1986). The mean true effect size is also estimated using a precisionweighted average of the observed effect sizes (Equation 7), but the variance of the observed effect sizes (and therefore, the weights) is now calculated on the basis of Equation 8. Raudenbush and Bryk (1985) showed that a metaanalysis is a special case of a multilevel analysis and that estimation procedures for multilevel models (usually maximum likelihood procedures) can be used to estimate the unknown parameters of the meta-analytic models. The different approaches may lead to different between-studies variance estimates, especially if the number of studies is small, and hence to slightly different estimates of the mean effect size and to different estimates of the standard errors of estimation. Van den Noortgate and Onghena (2003) showed that the DerSimonian and Laird and the maximum likelihood estimates are usually very similar and are, in general, to be preferred over the between-studies variance estimates proposed by Hedges and Olkin.

In both the fixed effects model (FEM) and the REM, study characteristics can be included as moderator variables, but the discussion in this article will be restricted to models without moderator variables.

\section{The Use of the Standardized Mean Difference in Meta-Analysis}

The use of the standardized mean difference in metaanalysis is appealing for different reasons, including the 
ease of interpretation: $d$ is equal to the difference between the means, after correction for different scaling. Indeed, if in different studies different but linearly equatable scales are used, dividing by the standard deviation makes the mean differences directly comparable. Moreover, $d$ is well known among many researchers, and formulas are widely available in the meta-analytic literature to convert a large range of effect size measures, test statistics, or $p$ values to $d$. Nevertheless, the standardized mean difference also has some drawbacks. One of them is its restricted applicability: $d$ can be used to compare two groups or to describe the effect of a dichotomous variable but cannot be used for continuous independent variables, in contrast with, for instance, the correlation coefficient.

Another problem, which has often been overlooked by meta-analysts, is the main focus of this article. The problem was pointed out by Hedges (1983; Hedges \& Olkin, 1985 ) in cases of homogeneous studies. As was discussed above, under the assumption of normally distributed populations with a common variance, $d$ gives an unbiased estimate of $\delta$ for each study. Consequently, the same is true for the unweighted mean of the observed effect sizes stemming from a random set of homogeneous studies. However, the weighted combination of $d$ s can be a biased estimate of $\delta$. This is the case in the common procedure, in which the inverses of the sampling variances are used as weights and the sampling variances are estimated by replacing $\delta$ with $d$ (Equation 6). This procedure implies that for large positive or negative $d$ s, the estimated sampling variance will be somewhat larger than that for small $d \mathrm{~s}$, resulting in a smaller weight. This means that, in general, the estimate of a nonzero $\delta$ based on a set of observed $d$ s is shrunken toward zero, resulting in a negative bias for estimating a positive $\delta$ and a positive bias for a negative $\delta$. Because the weights are less dependent on $\delta$ if the study sizes are large and because the variation of the observed effect sizes is smaller for a small true effect size (see Equation 6), we would expect that the problem is largest for small studies or studies estimating a large effect. In contrast, because the weights do not depend on the number of studies, we would expect that the bias is independent of the number of studies. In cases of heterogeneous studies, we would expect that the bias is even larger, because in this case the variance of the effect sizes is due not only to sampling variation, but also to the variance between true effect sizes. This means that in addition to the bias that is due to the fact that the sampling variance is not known but is estimated using the observed effect size, the estimate is biased because of the true between-studies variance. Finally, it can be deduced from Equations 7 and 8 that using an REM, instead of an FEM, can partially compensate for the bias, especially when the true variance is large: Because in the random effects procedure, not only the sampling variance, but also the true between-studies variance is taken into account in calculating the weights, the weights are less dependent on the sampling variances and are, thus, more equalized. In the next section, possible solutions for the problem of bias will be discussed.

\section{Improving Mean Delta Estimates}

As Hedges (1983) has pointed out, using weights that are independent of $\delta$ (e.g., $n_{\mathrm{E}} n_{\mathrm{C}} /\left(n_{\mathrm{E}}+n_{\mathrm{C}}\right)$ solves the problem of the bias in estimating a common $\delta$, but the resulting estimator is less efficient. Another, not frequently used, alternative is to employ a fixed estimate of $\delta$ for estimating the sampling variance in all studies. This can be an a priori estimate of $\delta$ or an estimate resulting from a metaanalysis. Because the estimates of the sampling variances are needed for a meta-analysis, Hedges and Olkin (1985) proposed an iterative meta-analysis. A meta-analysis is performed with weights estimated using the observed effect sizes, and the resulting estimate of the mean true effect size is used to estimate the weights for a second metaanalysis, and so on. Weights thus are updated in each successive meta-analysis. Note that Hunter and Schmidt (1990) proposed a similar (but noniterative) approach for combining correlation coefficients: The observed effect sizes are weighted with their corresponding reliabilities, which are estimated using the average correlation across studies.

The iterative procedure outlined by Hedges and Olkin (1985) may raise problems if the meta-analytic dataset contains heterogeneous studies, because in this case, the weighted mean of the effect sizes $\left(d_{+}\right)$gives an estimate of the mean effect size that may be relatively uninformative for the true effect sizes of the individual studies. In case studies that are strongly heterogeneous, estimating the sampling variance by replacing $\delta_{j}$ with the estimate of the mean effect size does not seem to be recommended, whereas the use of the observed effect sizes may be a better choice.

Empirical Bayes effect size estimates (Rubin, 1981) are optimally weighted combinations of these two kinds of estimates, with weights depending on the sampling variation (and thus on the study sizes) and on the variance between study effect sizes:

$$
d_{\mathrm{EB}}=\lambda_{j} d_{j}+\left(1-\lambda_{j}\right) d_{+},
$$

where

$$
\lambda_{j}=\hat{\sigma}^{2}(\delta) /\left[\hat{\sigma}^{2}(\delta)+\hat{\sigma}^{2}\left(d_{j} \mid \delta_{j}\right)\right]
$$

In Equation 9, it can be seen that if the (estimated) true variance is large, less weight will be given to the estimate of the overall effect size. Indeed, the more similar the effect sizes being estimated, the better the estimate of the mean effect size functions as an estimate of the effect size in a certain study. Not only the variance between effect sizes, but also the study sizes are important in choosing an estimate of the individual effect sizes: For a large study, the effect size estimate of that study is relatively reliable, and there is less reason to lean on the estimates from other studies. Therefore, in large studies, a relatively large weight is given to the observed effect size from that study. Although empirical Bayes estimates are not unbiased, the $M S E$ is reduced.

Therefore, using the empirical Bayes estimates for estimating the sampling variances seems to be an interesting 
alternative to using the weighted mean of effect sizes. Because the empirical Bayes estimates are a result of the meta-analysis, an iterative procedure can be followed, in which the sampling variance estimates and the mean effect size estimate are updated in turn. In cases in which the (estimated) variance between effect sizes is zero, the empirical Bayes estimates are equal to the mean effect size estimate, making this procedure equivalent to the iterative procedure outlined by Hedges and Olkin (1985) for homogeneous studies. In the other extreme situation, in cases in which the estimated variance and/or the study sizes tend to infinity, the empirical Bayes estimates are equal to the observed effect size estimates, making this procedure equivalent to the ordinary procedure, replacing the true effect sizes by the observed ones.

In the following, the results of a small simulation study will be reported. The first purpose of the study is to explore further the extent of the problem of estimating the overall effect size, using the ordinary procedure. Because the unbiasedness is often of less importance than the $M S E$, we will also take the $M S E$ into account. A second purpose is to compare the ordinary procedure, the iterative procedure of Hedges and Olkin (1985), and the alternative procedure in which empirical Bayes estimates are used.

\section{A Simulation Study}

Meta-analytic datasets were simulated with the following characteristics: group sizes $\left(n_{\mathrm{E}}=n_{\mathrm{C}}=n\right)$ approximately equal to 10,25 , or 50 ; number of studies $(k)$ equal to 5,10 , or 50 ; (mean) true effect sizes, $\delta$, equal to $0.5,1$, or 1.5; and finally, variance between effect sizes, $\sigma^{2}(\delta)$, equal to $0,0.1$, or 0.2 . These values are representative for results that have often occurred in practice or have been used in other simulation studies (e.g., Hedges, Cooper, \& Bushman, 1992; Hedges \& Vevea, 1998). For each of these 81 combinations, 5,000 meta-analytic datasets were simulated using MLwiN (Goldstein et al., 1998). For each study in each dataset, an effect size $\delta_{j}$ was drawn out of a normal distribution with a mean of $\delta$ and a variance of $\sigma^{2}(\delta)$. To define the group sizes in this study, a value was drawn out of a uniform distribution with bounds of $0.8 n$ and $1.2 n$, before being rounded. For both groups, this number of observations was drawn from the control condition population distribution, $N\left(-\delta_{j} / 2,1\right)$, and from the experimental condition population distribution, $N\left(\delta_{j} / 2,1\right)$. The "observed" effect size for the study was calculated on the basis of these raw data in the usual way (using Equations 3 and 4 ).

For each dataset, we used an FEM to estimate the mean of the true effects of the studies included in the dataset, as well as an REM to estimate the mean of the population of true effects from which the true effects were sampled. For both the fixed effects and the random effects procedure, these parameters were estimated using the RIGLS algorithm implemented in MLwiN, resulting in restricted maximum likelihood estimates for the unknown parameters. The estimate of $\delta$ was a weighted mean of the observed effect sizes. The weights were estimated in three ways: with the observed effect sizes $(d)$, the weighted average of the observed effect sizes $\left(d_{+}\right)$, and the empirical Bayes estimates of the true effect sizes $\left(d_{\mathrm{EB}}\right)$. For the approaches in which the overall effect size estimate or the empirical Bayes estimates were used to estimate the weights, we used 10 iterations. To evaluate the results, we estimated the bias of the estimates for each combination, using the estimates for the 5,000 meta-analytic datasets in this combination. This was done by averaging the estimation errors, which are the differences between the estimates and the true parameter values. Note that in cases in which an FEM was used, the parameter that was estimated was the mean of the true effects of the studies included in the dataset. In cases in which an REM was used, the estimated parameter was the mean of the population of true effects. Moreover, we estimated the variance of the estimation errors and the $M S E$ around the true parameter value. The results are found in Table 1 (FEM) and Table 2 (REM). To obtain parsimonious tables, we report only the estimated bias and the $M S E$ and leave out the results for the intermediate effect size $(\delta=1)$ and the variance $\left[\sigma^{2}(\delta)=0.1\right]$. Although not reported, the variance estimates can easily be obtained by subtracting the squared bias estimate from the estimated $M S E$, using the following equation:

$$
\text { variance }=M S E-(\text { bias })^{2} \text {. }
$$

The ordinary procedure. Let us look first at the bias in estimating the mean $\delta$ for the FEM if the observed effect sizes are used to estimate the sampling variances. As was expected, the bias is smaller for increasing $n$. Moreover, the bias increases with increasing true variance and for larger true mean $\delta$ s. Unexpectedly, the bias seems to increase slightly for an increasing number of studies. This can be explained by noting that the larger the number of studies in a meta-analytic dataset, the more unlikely it is that, in all the studies, similar effect sizes are reported, whereas it is especially in cases in which dissimilar results are combined that the bias is visible. This is most obvious in the extreme case in which there is only one study in a meta- analytic dataset: The effect size estimate of a single study is unbiased.

For the REM, the bias is smaller than that for the FEM, as we expected, because taking into account the variation between true effect sizes equalizes the weights. This is especially true if this true variance is large. Moreover, $k, n$, and $\delta$ seem to have an effect on the bias similar to that for the FEM. Although the effect of the variance is in the same direction as that in the FEM, the effect is strongly reduced: As in the FEM, an increased variance means an increased difference in the estimated sampling variances and, thus, an increase of the bias, but the effect on the weights is largely compensated for by the equalizing effect of the true variance on the weights.

As was expected, the variance of the estimation errors decreases with increasing $n$ and increases slightly with increasing mean $\delta$ (this is most easily seen in Table 3 , although after some calculation, the values in Table 3 could be deduced from Tables 1 and 2). As a result, the MSE is af- 
Table 1

Bias $(\times 10,000)$ and $M S E(\times 10,000)$ for the Fixed Effects Model When the Mean Effect Size Is Estimated, With Weights Based on the Observed Effect Sizes $(d)$, the Weighted Average of the Observed Effect Sizes $\left(d_{+}\right)$, or the Empirical Bayes Estimates $\left(d_{\mathrm{EB}}\right)$

\begin{tabular}{|c|c|c|c|c|c|c|c|c|}
\hline \multirow[b]{2}{*}{$\delta$} & \multirow[b]{2}{*}{$n$} & \multirow[b]{2}{*}{$k$} & \multicolumn{3}{|c|}{ Variance 0} & \multicolumn{3}{|c|}{ Variance 0.2} \\
\hline & & & $d$ & $d_{+}$ & $d_{\mathrm{EB}}$ & $d$ & $d_{+}$ & $d_{\mathrm{EB}}$ \\
\hline \multicolumn{9}{|c|}{ Bias } \\
\hline \multirow[t]{9}{*}{0.5} & 10 & 5 & -214 & -14 & -65 & -389 & -28 & -211 \\
\hline & & 10 & -222 & 2 & -33 & -379 & 28 & -155 \\
\hline & & 50 & -248 & -6 & -16 & -439 & -8 & -188 \\
\hline & 25 & 5 & -70 & 11 & -10 & -267 & -18 & -193 \\
\hline & & 10 & -92 & -1 & -17 & -279 & 1 & -194 \\
\hline & & 50 & -99 & -2 & -8 & -309 & -10 & -217 \\
\hline & 50 & 5 & -25 & -16 & -26 & -205 & 9 & -168 \\
\hline & & 10 & -30 & 15 & 7 & -245 & 11 & -203 \\
\hline & & 50 & -44 & 0 & -4 & -263 & -6 & -219 \\
\hline \multirow[t]{10}{*}{1.5} & 10 & 5 & -568 & 47 & -111 & $-1,027$ & -15 & -498 \\
\hline & & 10 & -712 & 38 & -138 & $-1,133$ & -14 & -474 \\
\hline & & 50 & -734 & 6 & -28 & $-1,222$ & -9 & -463 \\
\hline & 25 & 5 & -244 & -1 & -67 & -664 & -12 & -445 \\
\hline & & 10 & -285 & -17 & -63 & -739 & 6 & -478 \\
\hline & & 50 & -295 & 0 & -19 & -810 & -2 & -527 \\
\hline & 50 & 5 & -120 & 1 & -31 & -545 & 0 & -432 \\
\hline & & 10 & -133 & 2 & -22 & -625 & 6 & -496 \\
\hline & & 50 & -148 & -1 & -12 & -671 & 0 & -533 \\
\hline & \multicolumn{8}{|c|}{$M S E$} \\
\hline \multirow[t]{9}{*}{0.5} & 10 & 5 & 402 & 435 & 424 & 407 & 453 & 424 \\
\hline & & 10 & 204 & 222 & 218 & 202 & 222 & 206 \\
\hline & & 50 & 44 & 42 & 42 & 58 & 46 & 46 \\
\hline & 25 & 5 & 163 & 169 & 167 & 167 & 173 & 167 \\
\hline & & 10 & 78 & 81 & 80 & 88 & 88 & 86 \\
\hline & & 50 & 17 & 16 & 16 & 25 & 17 & 21 \\
\hline & 50 & 5 & 85 & 86 & 85 & 89 & 89 & 88 \\
\hline & & 10 & 42 & 43 & 42 & 49 & 46 & 48 \\
\hline & & 50 & 8 & 8 & 8 & 15 & 9 & 13 \\
\hline \multirow[t]{9}{*}{1.5} & 10 & 5 & 514 & 555 & 525 & 584 & 568 & 536 \\
\hline & & 10 & 295 & 283 & 273 & 365 & 279 & 281 \\
\hline & & 50 & 102 & 56 & 54 & 197 & 57 & 75 \\
\hline & 25 & 5 & 208 & 213 & 208 & 259 & 224 & 240 \\
\hline & & 10 & 110 & 107 & 105 & 157 & 107 & 128 \\
\hline & & 50 & 29 & 21 & 21 & 87 & 22 & 50 \\
\hline & 50 & 5 & 104 & 104 & 104 & 143 & 110 & 133 \\
\hline & & 10 & 53 & 53 & 53 & 95 & 54 & 81 \\
\hline & & 50 & 12 & 10 & 10 & 57 & 11 & 40 \\
\hline
\end{tabular}

fected in a similar way (Tables 1 and 2). If an REM is used, increasing the true between-studies variance inflates the variance of the estimates of the overall effect and, consequently, also the MSE (Tables 2 and 3). If an FEM is used, the variance of the estimation errors is hardly affected (Table 3). Indeed, when using an FEM, one estimates the mean of the true effect sizes of the specific studies included in the dataset, and the deviation of the estimate from this mean is affected only by the sampling variance within studies. However, as we saw before, increasing the true between-studies variance means an increased bias, especially for a large $\delta$, resulting in an increased MSE (Table 1).

With increasing $k$, the $M S E$ is affected more by the decrease in the variance than by the increase in bias, resulting in a decreasing $M S E$.
Although in general, the bias is smaller if an REM is used, the variance and the $M S E$ are larger, unless the true betweenstudies variance is zero. In the REM, the variation of the observed effect sizes and of the weighted means around the true parameter values is indeed affected not only by sampling variation, but also by the variation in true effect sizes. A reduced $M S E$ cannot, however, be used as an argument for using an FEM. Rather, the choice of the model must depend on the research interests. When using an FEM, the researcher is interested in the mean of the effects investigated in these specific studies; when an REM is used, the main focus is on the mean effect of the population from which the studies are regarded as a random sample.

Finally, note that the contribution of the bias to the MSE is often small. For instance, for the FEM, the bias is -0.0214 
Table 2

Bias $(\times 10,000)$ and $M S E(\times 10,000)$ for the Random Effects Model

When the Mean Effect Size Is Estimated, With Weights Based

on the Observed Effect Sizes $(d)$, the Weighted Average

of the Observed Effect Sizes $\left(d_{+}\right)$, or the Empirical Bayes Estimates $\left(d_{\mathrm{EB}}\right)$

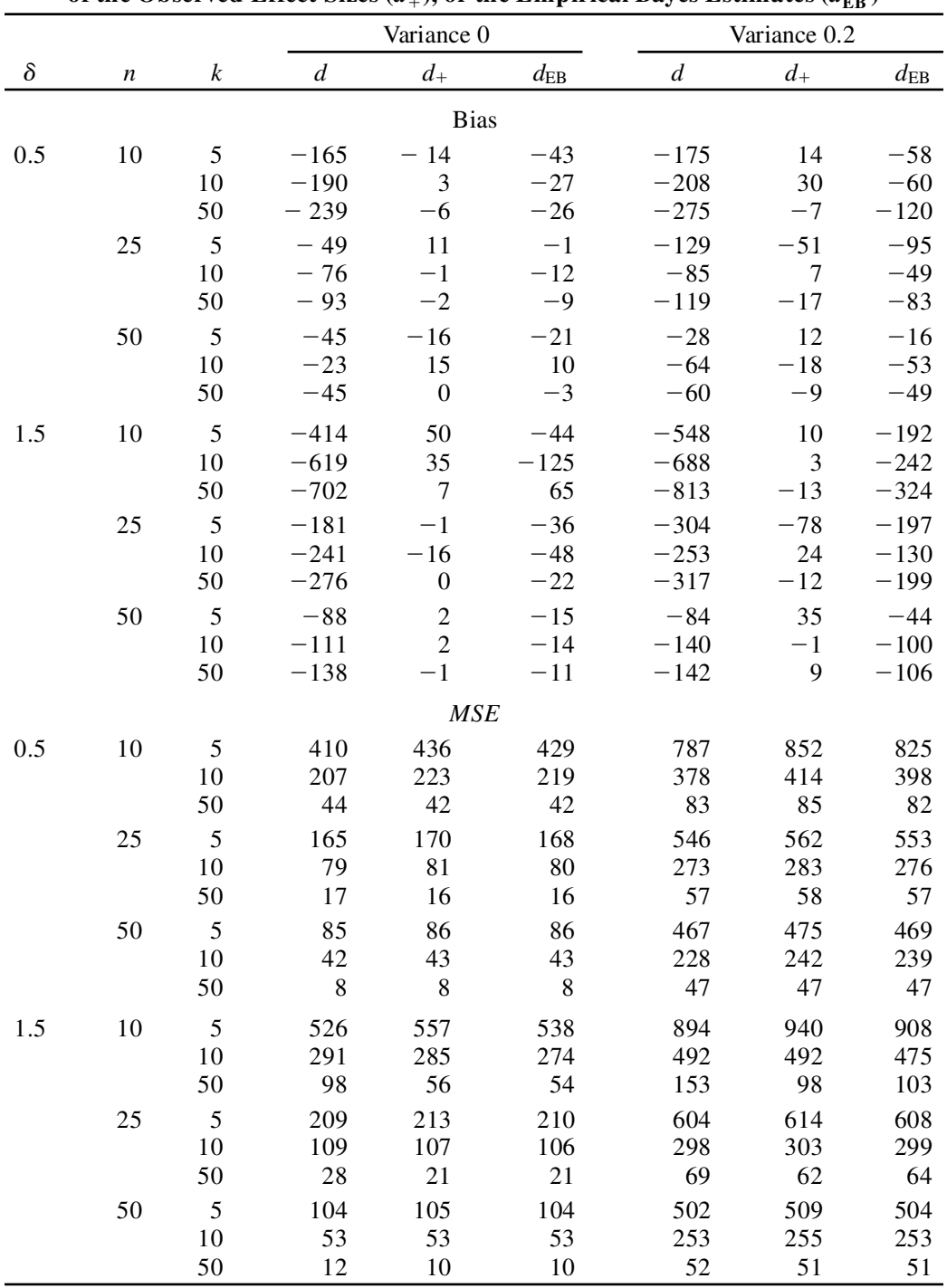

if the meta-analytic dataset consists of five studies with group sizes equal to 10 and true overall effect size equal to 0.5 . This means that from the $M S E$ of 0.0482 , only $0.0005\left(=-0.0214^{2}\right)$ is due to bias (Equation 12). The bias will be relatively large, as compared with the $M S E$, only when all three of the following are large: $k$, the true variance, and $\delta$.

Comparing the three approaches. In Tables 1 and 2, it can be seen that the estimates of the bias for the approach in which $d_{+}$is used are small and not systematic. This is true for FEMs as well as for REMs. This corresponds with our expectations that using $d_{+}$results in an elimination of the bias. The results of the empirical Bayes approach are situated in between. Using the empirical
Bayes estimates thus reduces the bias, without, however, eliminating the bias.

Concerning the variances of the estimates (which are not reported here), using $d_{+}$generally results in a larger variance of the estimates, as compared with the ordinary procedure. This seems to be true for all combinations and for REM as well as for FEMs. The results of the empirical Bayes procedure are again situated between the two other procedures. For large $k$, however, the variances in the estimates of the three approaches are comparable. The positive effect on the variance of the estimates of increasing $k$, indeed, is more pronounced in the $d_{+}$procedure and weakest in the ordinary procedure. The effect of $n$, the true variance, and $\delta$ on the variance of the estimates is similar 
Table 3

Variance $(X 10,000)$ of the Estimation Errors for the Fixed Effects Model (FEM) and the Random Effects Model (REM) Using the Observed Effect Sizes $(d)$

\begin{tabular}{|c|c|c|c|c|c|c|c|c|}
\hline \multirow[b]{2}{*}{$\delta$} & \multicolumn{2}{|c|}{ Variance 0} & \multicolumn{2}{|c|}{ Variance 0.1} & \multicolumn{2}{|c|}{ Variance 0.2} & \multicolumn{2}{|c|}{ Overall } \\
\hline & FEM & REM & FEM & REM & FEM & REM & FEM & REM \\
\hline 0.5 & 116 & 117 & 116 & 219 & 122 & 319 & 118 & 219 \\
\hline 1 & 130 & 132 & 142 & 237 & 162 & 349 & 145 & 237 \\
\hline 1.5 & 159 & 159 & 182 & 269 & 216 & 368 & 186 & 265 \\
\hline Overall & 135 & 136 & 147 & 242 & 167 & 344 & 149 & 240 \\
\hline
\end{tabular}

in the three approaches. These results can again be explained by the dependence of the weights and the effect sizes. If each study's estimate of $d$ is used to compute the weights, outlying positive or negative observed effects sizes receive a relatively low weight and, hence, do not have a large influence on the results. If, on the contrary, the mean effect size is used to calculate the weights, outlying effect sizes are weighted as much as moderate effect sizes. Thus, if the mean effect size is used, the effect of outliers can be considerable, especially if the number of effect sizes is small, explaining the larger variance in the estimates.
Probably the most important criterion in the comparison is the $M S E$. Although using $d_{+}$generally results in smaller bias and using $d$ in smaller variance of the estimates, the picture is somewhat more complicated for the MSE. If the meta-analytic dataset consists of a small number of studies, the $M S E$ is often smallest if the observed effect sizes are used to estimate the sampling variances and largest if the $d_{+}$is used. We saw that in the ordinary procedure (and to a smaller degree, in the empirical Bayes procedure), the bias increases with increasing $k$. Meanwhile, in the ordinary procedure (and to a smaller degree, in the empirical

Table 4

Mean Square Error $(\times 10,000)$ for the Fixed Effects Model $($ FEM) and the Random Effects Model (REM) When the Mean Effect Size Is Estimated, With Weights Based on the Observed Effect Sizes $(d)$, the Weighted Average of the Observed Effect Sizes $\left(d_{+}\right)$, or the Empirical Bayes Estimates $\left(d_{E B}\right)$

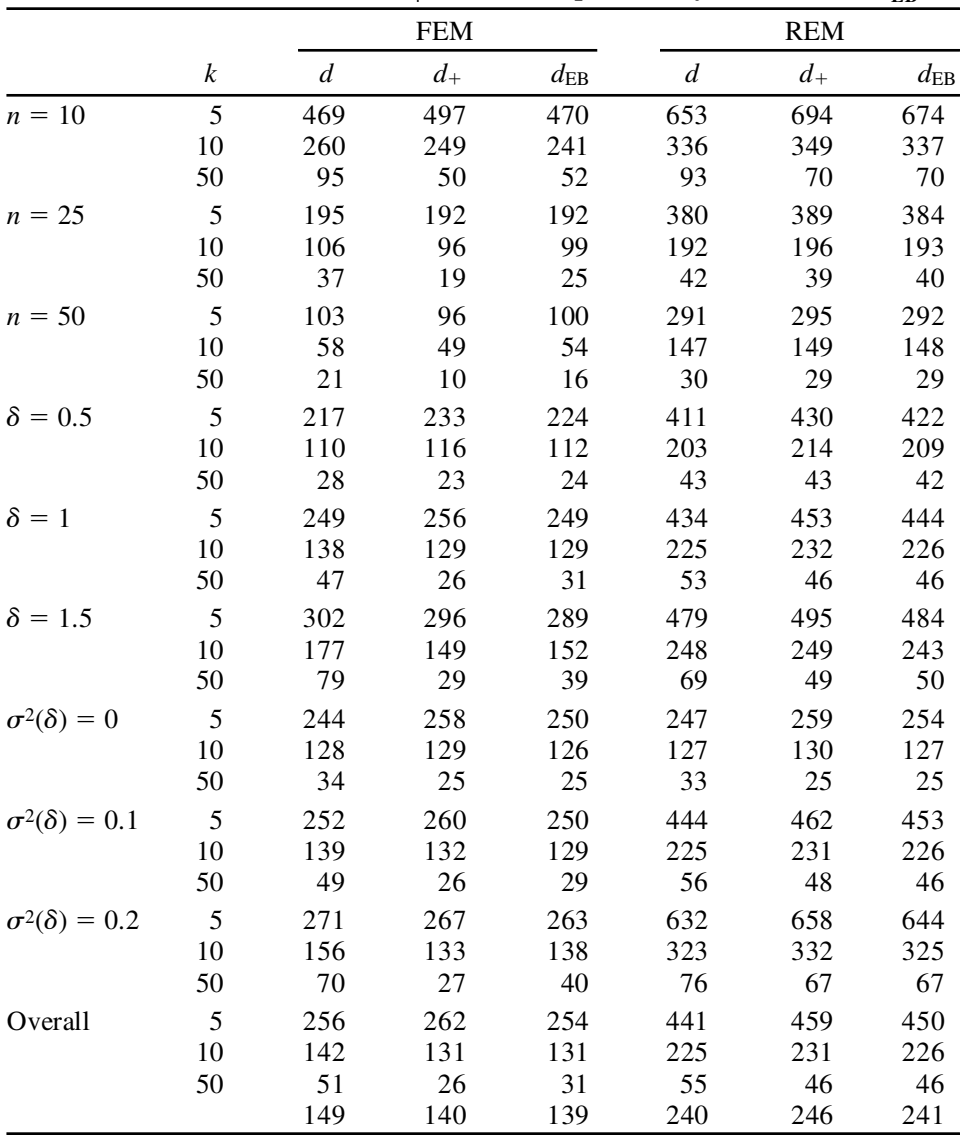


Bayes procedure), the variance decreases more slowly than in the $d_{+}$procedure. The result is that whereas for a small number of studies the MSE is smallest if the observed effect sizes are used, for a large number of studies the $d_{+}$procedure gives the best results. In between, for a moderate number of studies, one can often profit from the empirical Bayes procedure. This trend can be seen in Table 4.

A complicating factor is that the number of studies in the meta-analytic dataset for which each of the three procedures performs the best depends on other characteristics of the meta-analytic dataset. In Table 4, it can be seen that the number of studies for which the empirical Bayes or the $d_{+}$approach is better than the ordinary approach is smaller if $n$ is larger: If, for instance, an FEM is used when $n=10$, the ordinary procedure performs the best if $k=5$, closely followed by the empirical Bayes approach; for $k=$ 10 , the empirical Bayes approach is the best; for $k=50$, the $d_{+}$procedure has become the best procedure. When $n=50$, on the contrary, the $d_{+}$procedure yields the best results, even for a very small number of studies. The effect of $n$ is similar for an REM. Similarly, it can be concluded, again for both FEMs and REMs, that the number of studies for which the empirical Bayes or the $d_{+}$approach is better than the ordinary approach is smaller if $\delta$ is larger. In addition, we found that the number of studies for which the empirical Bayes or the $d_{+}$approach is better than the ordinary approach is smaller if the variance is larger. This, however, seems to be true only for the FEM. Finally, we conclude that the number of studies for which the empirical Bayes or the $d_{+}$approach is better than the ordinary approach is smaller if an FEM is used.

\section{Conclusions}

In the article, we focused on the problem of estimating the mean effect size. As has been pointed out before (e.g., Hedges, 1983), the precision-weighted average of observed effect sizes resulting from homogeneous studies, with optimal weights estimated using the observed effect sizes, gives a biased estimate of the mean effect. Although we found that the problem of bias is even larger when the studies are heterogeneous and that the bias does not disappear with increasing $k$, but even increases, the results of our simulation study suggest that the bias is often relatively small, taking into consideration the variance of the estimates. Only when $k$, the true variance, and $\delta$ are large is a substantial part of the MSE due to bias.

In the article, we have proposed an alternative approach: The overall effect size is still estimated using a precisionweighted mean of the observed effect sizes, but this time, empirical Bayes estimates of the true effect sizes are used to estimate the precision. The approach is compared with the ordinary approach and with an iterative approach previously presented by Hedges and Olkin (1985). Although the bias disappears when the iterative approach proposed by Hedges and Olkin is used and the variance of the estimates is usually smallest when the ordinary approach is used, there is no uniformly best approach regarding the MSE.
An interesting result is that the iterative approach of Hedges and Olkin (1985), using the overall effect size estimate to estimate the weights, may even exacerbate the problem by increasing the $M S E$. Still, this procedure often means an improvement if the number of studies is large. This is true even when study results are heterogeneous and the overall effect size estimate, therefore, is only a rough estimate of the individual effect sizes. In general, if the meta-analytic dataset consists of a small number of studies, the ordinary approach usually performs better; for a moderately sized dataset, the empirical Bayes approach often yields the best results; for a large meta-analytic dataset, the iterative approach of Hedges and Olkin usually can be recommended. This is true for both REMs and FEMs. However, the sizes of the meta-analytic dataset for which each of the three procedures performs the best depend on other characteristics of the meta-analytic dataset. The number of studies for which the empirical Bayes or the $d_{+}$ approach is better than the ordinary approach is larger if $\delta$ is small, if $n$ is small, and if an REM is used. For the FEM, this number gets smaller with increasing true variance.

\section{REFERENCES}

COHE N, J. (1969). Statistical power analysis for the behavioral sciences. New York: Academic Press.

DERSIMOnian, R., \& LAIRD, N. (1986). Meta-analysis in clinical trials. Controlled Clinical Trials, 7, 177-188.

GLASS, G. V. (1976). Primary, secondary, and meta-analysis of research. Educational Researcher, 5, 3-8.

GLASS, G. V. (1977). Integrating findings: The meta-analysis of research. Review of Research in Education, 5, 351-379.

GLASs, G. V., MACGAW, B., \& SMITH, M. L. (1981). Meta-analysis in social research. Beverly Hills, CA: Sage.

Goldstein, H., Rasbash, J., Plewis, I., Draper, D., Browne, W., YAng, M., Woodhouse, G., \& HeAly, M. (1998). A user's guide to MLwiN. London: University of London, Multilevel Models Project.

HeDGES, L. V. (1981). Distribution theory for Glass's estimator of effect size and related estimators. Journal of EducationalStatistics, 6, 107-128.

HedGES, L. V. (1982). Estimation of effect size from a series of independent experiments. Psychological Bulletin, 92, 490-499.

Hedges, L. V. (1983). A random effects model for effect sizes. Psychological Bulletin, 93, 388-395.

Hedge S, L. V., Cooper, H., \& Bushman, B. J. (1992). Testing the null hypothesis in meta-analysis: A comparison of combined probability and confidence interval procedures. Psychological Bulletin, 111, 188-194.

HedGe S, L. V., \& OL KIN, I. (1985). Statistical methods for meta-analysis. Orlando, FL: Academic Press.

HedGeS, L. V., \& VEVEA, J. L. (1998). Fixed- and random-effects models in meta-analysis. Psychological Methods, 3, 486-504.

HunTER, J. E., \& SCHMIDT, F. L. (1990). Methods ofmeta-analysis: Correcting error and bias in research findings. London: Sage.

HunTER, J. E., SCHMIDT, F. L., \& JACKSON, G. B. (1982). Meta-analysis: Cumulating findings across research. Beverly Hills, CA: Sage.

RAudenbush, S. W., \& BRYK, A. S. (1985). Empirical Bayes metaanalysis. Journal of Educational Statistics, 10, 75-98.

Rosenthal, R. (1991). Meta-analytic procedures for social research. Newbury Park, CA: Sage.

RUBIN, D. B. (1981). Estimation in parallel randomized experiments. Journal of Educational Statistics, 6, 377-400.

Van den Noortgate, W., \& Onghena, P. (2003). Multilevel metaanalysis: A comparison with traditional meta-analytical procedures. Educational \& Psychological Measurement, 63, 765-790.

(Manuscript received March 5, 2002; revision accepted for publication January 14, 2003.) 